Reprod. Nutr. Dévelop., 1986, 26 (5 A), 1115-1135.

\title{
Evolution du tractus uro-génital chez les Pleurodèles chimères à corps double. Effet de position dans les interactions entre glandes génitales de sexe différent.
}

\author{
Ch. HOUILLON, C. DOURNON
}

Laboratoire de Biologie animale, Université $P$. et M. Curie, 9 quai Saint-Bernard, 75252 Paris Cedex 05, France.

avec la collaboration technique de Mme SZMUTYK pour les élevages et de Mr DESROSIERS pour l'illustration.

Summary. Development of the urogenital tract in double-bodied pleurodele chimeras : position effect on interactions between gonads of different sex.

Chimeric grafts of the embryos of the urodele amphibian (Pleurodeles waltiii Michah.) produced viable, double-bodied animals having two pairs of genital glands of the same sex [homosexual chimeras : anterior testes - posterior testes $\left(\sigma^{\circ} \leftrightarrow \sigma^{\circ}\right)$ and anterior ovaries - posterior ovaries ( $९ \leftrightarrow ९)$ ] or of different sex [(heterosexual chimeras :

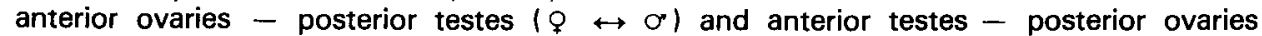
$\left(\sigma^{\circ} \leftrightarrow\right.$ \&)]. 115 chimeras aged 5 months to 15 years were analysed. Gonadal development in a same animal was observed at different times by exploratory laparotomy. The testes of heterosexual chimeras were removed in order to prevent their inhibitory effect on the ovaries (freemartin effect). follows :

The 115 experimental animals were classifed according to gonadal development as

-47 ○ $\leftrightarrow \sigma^{*}$ chimeras $(41 \%)$,

$-26 \% \leftrightarrow \%$ chimeras $(23 \%)$,

-13 \& $\leftrightarrow$ o chimeras $(11 \%)$,

- 29 o $\leftrightarrow$ \% chimeras $(25 \%)$.

As a rule, $\sigma^{\circ} \leftrightarrow \sigma^{\prime}$ chimeras only developed three normal testes. The fourth testis, always situated in the anterior part of the chimera, remained vestigial; nevertheless, it could attain a normal size if the other three testes were removed. $Q \leftrightarrow \leftrightarrow$ chimeras usually had four ovaries ; the times of vitellogenesis and of sexual maturity were synchronous in all four ovaries. The anterior ovaries of $Q \leftrightarrow \sigma^{*}$ chimeras were always inhibited and the posterior testes were always normal. $\sigma^{*} \leftrightarrow q$ chimeras always had normal anterior testes but their posterior ovaries were often weakly inhibited.

The high number of $0^{*} \leftrightarrow \sigma^{*}$ chimeras and the low number of $९ \leftrightarrow \leftrightarrow \quad$ ones could be due to a phenotypic reversal of the anterior ovaries in some of the $\leftrightarrow \leftrightarrow \circ$ chimeras. The number of $\sigma \leftrightarrow \leftrightarrow$ chimeras (corresponding to the theoretical number) suggests that the phenotypic sex of the posterior ovaries was not reversed.

The freemartin effect in double-bodied heterosexual chimeras depended on the location of the ovaries in relation to the testes. The effect was more significant on the anterior ovaries when posterior testes were present. No or weak freemartin effect was observed when the posterior ovaries developed facing the anterior testes; in about $60 \%$ 
of cases, a reciprocal inhibition or an equilibrium between gonads of different sex occurred. The equilibrium could be continuously maintained (hermaphroditic chimeras) or interrupted after several years. In the last case, we observed preferential development of either the anterior testes (late freemartin effect) or the posterior ovaries (no freemartin effect).

\section{Introduction.}

L'analyse des interactions entre glandes génitales de sexe différent, chez les Amphibiens, a fait l'objet de nombreux travaux expérimentaux ayant trait à la parabiose et aux greffes embryonnaires (revue dans Foote, 1964 ; Gallien, 1973 ; Collenot et al., 1977). Notre contribution personnelle concerne la technique originale de greffe en chimère embryonnaire qui offre de multiples avantages par rapport aux techniques précédentes (Houillon et al., 1971, 1973, 1974). Entre autres, la viabilité des animaux chimères est pratiquement la même que celle des animaux témoins alors que les paires parabiontiques sont délicates à élever. Par rapport à la greffe embryonnaire d'ébauches gonadiques qui rétablit difficilement les connexions anatomiques au niveau du tractus uro-génital, la greffe en chimère permet l'édification d'un tractus uro-génital parfaitement fonctionnel pour l'émission des gamètes.

La plupart des auteurs s'accordent pour reconnaître que chez les Amphibiens, la présence de testicules perturbe profondément le développement des ovaires à la manière de l'effet free-martin chez les Mammifères (Jost et al., 1975). Dans le cas plus particulier du Pleurodèle, l'inhibition ovarienne n'aboutirait qu'exceptionnellement à l'inversion du phénotype gonadique femelle (Collenot et al., 1977).

L'originalité du présent travail en plus du fait qu'il concerne des chimères à corps double, réside dans les points suivants : le nombre important d'animaux analysés (115), leur âge (de 5 mois à 15 ans), la pratique systématique des laparotomies exploratrices et l'ablation des testicules afin de lever l'action inhibitrice qu'ils exercent sur l'évolution ovarienne. II ressort de cette étude que les interactions entre testicules et ovaires dépendent avant tout de leur position relative : l'inhibition ovarienne n'est pas un phénomène général et l'inversion du phénotype ovarien est assez fréquente.

\section{Matériel et méthodes.}

La technique de la greffe embryonnaire en chimère chez les Amphibiens Urodèles, d'abord mise en œuvre pour obtenir des chimères xénogéniques (Houillon, 1964a, b, 1965), a été précisée par ailleurs pour obtenir les chimères à corps simple et à corps double chez le Pleurodèle (Charlemagne et Houillon, 1974), puis chez le Triton alpestre (Houillon, 1977). Le génotype sexuel des embryons étant inconnu au moment de l'intervention, les associations réalisées peuvent être soit 
homosexuées (entre embryons de même sexe), soit hétérosexuées (entre embryons de sexe différent) (fig. 1).

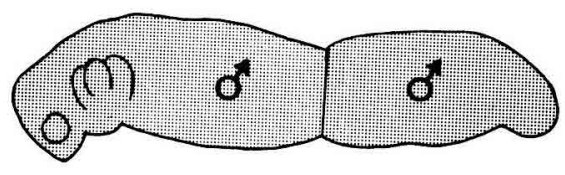

Chimères homosexuées

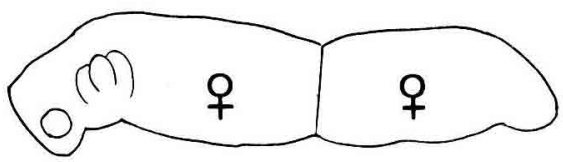

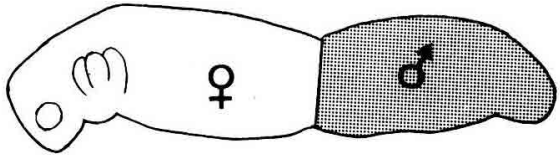

Chimères hétérosexuées

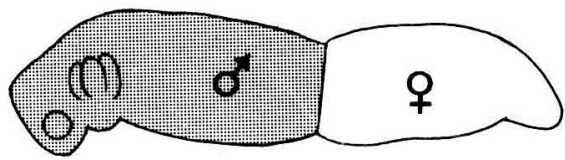

FIG. 1. - Associations entre embryons de même sexe et de sexe différent chez les Pleurodèles chimères à corps double.

Le sexe des glandes génitales se reconnaît macroscopiquement et toujours sans ambiguïté lors d'une laparotomie ou à la dissection sauf pour les glandes antérieures dans le cas particulier d'une association femelle antérieure-mâle postérieure. La désignation d'une chimère est donnée en premier par le sexe de sa partie antérieure et ensuite par le sexe de sa partie postérieure lorsque la nature des gonades est identifiée avec certitude. Ainsi, la désignation $q \leftrightarrow$ correspond à une chimère homosexuée partie antérieure femelle-partie postérieure femelle. La désignation $\sigma^{*} \leftrightarrow$ Q correspond à une chimère hétérosexuée partie antérieure mâle-partie postérieure femelle. La désignation particulière $(\varphi) \leftrightarrow \sigma$ signifie que la partie antérieure est considérée en première approximation, mais sans aucune certitude, comme femelle ; lorsque cette certitude est acquise, la chimère est désignée $Q \leftrightarrow O^{*}$.

Les caractères externes de la maturité sexuelle mâle qui apparaissent vers l'âge d'un an chez les Pleurodèles témoins, correspondent aux callosités sur les membres antérieurs et à la turgescence du cloaque. Les laparotomies exploratrices sont pratiquées en vue d'observer le tractus uro-génital d'un animal particulier à un âge donné pour ensuite pouvoir interpréter son évolution quelques années, voire une dizaine d'années après. L'intervention s'effectue sous la loupe binoculaire après anesthésie de l'animal. II est souvent nécessaire de faire une longue incision sur chaque flanc de façon à observer en même temps le tractus urogénital antérieur et le tractus uro-génital postérieur. L'ablation des testicules a pour objet de lever l'inhibition qu'ils exercent sur les ovaires (effet free-martin) afin de suivre ensuite l'évolution de ces gonades dont l'organogenèse initiale a été perturbée. L'ablation d'un seul testicule est souvent utile pour permettre le développement d'un testicule vestigial ou demeuré à l'état vestigial ; le terme " vestigial » signifie ici qu'il s'agit d'une gonade mâle très réduite dès sa formation. La castration testiculaire, souvent concomitante d'une laparotomie, n'offre pas de difficultés majeures. Une fois prélevées à l'aide d'un thermocautère, les gonades 
mâles sont fixées à des fins histologiques ou conservées comme contrôle d'un développement ultérieur. La suture des incisions est faite avec précaution de façon à n'entraîner aucune adhérence des viscères aux parois internes, ce qui rend souvent délicates une seconde laparotomie et la dissection. Certaines incisions nécessairement très longues pour des animaux à corps double, demandent parfois jusqu'à 12 points de suture sur chaque flanc. Cependant, aucune mortalité particulière post-opératoire n'a été enregistrée à la suite des laparotomies et des castrations.

\section{Résultats et interprétations.}

Le nombre d'animaux consignés dans le tableau 1 pour les 4 associations, correspond à tous les Pleurodèles chimères à corps double obtenus au laboratoire depuis deux décennies. Leur âge au moment de la dissection indique clairement que ces animaux ont une longévité comparable à celle des animaux témoins. Les chimères ne sont prises en compte qu'à partir de 5 mois, soit au moins un mois après la métamorphose ; il $\mathrm{y}$ a davantage $\mathrm{d}$ 'animaux âgés de 10 années et plus (19 cas) que d'animaux âgés de moins d'une année (10 cas).

Les résultats rapportés auparavant prennent désormais toute leur valeur et peuvent se résumer comme suit avant d'être détaillés pour chaque association :

- les chimères $\sigma^{\prime \prime} \leftrightarrow \sigma^{\prime} n^{\prime}$ ont en général que 3 testicules d'aspect normal et dans le même état de maturité. Les testicules postérieurs sont toujours bien développés ; I'un des testicules antérieurs souvent vestigial, évolue rapidement en testicule normal après l'ablation des 3 autres ;

- les chimères $९ \leftrightarrow ९$ ont toujours 2 ovaires postérieurs d'aspect normal et dans la plupart des cas les 2 ovaires antérieurs sont également bien développés. La vitellogenèse et la maturité sexuelle interviennent en même temps pour tous les ovaires ;

TABLEAU 1 :

Age des Pleurodèles chimères à corps double.

\begin{tabular}{|c|c|c|c|c|c|}
\hline Association & $\begin{array}{l}5 \text { mois } \\
\text { à moins de } \\
12 \text { mois }\end{array}$ & $\begin{array}{c}1 \text { an } \\
\text { à moins de } \\
4 \text { ans }\end{array}$ & $\begin{array}{l}4 \text { ans } \\
\text { à moins de } \\
10 \text { ans }\end{array}$ & $\begin{array}{l}10 \text { ans } \\
\text { et plus }\end{array}$ & total \\
\hline$\sigma^{*} \leftrightarrow \sigma^{*}$ & 4 & 10 & 1 & 4 & 19 \\
\hline$Q \leftrightarrow Q$ & 3 & 15 & 7 & 1 & 26 \\
\hline${ }^{*}(Q) \leftrightarrow \sigma^{*}$ & - & 15 & 15 & 11 & 41 \\
\hline$\sigma^{\circ} \leftrightarrow q$ & 3 & 15 & 8 & 3 & 29 \\
\hline Total & 10 & 55 & 31 & 19 & 115 \\
\hline
\end{tabular}

* Les nombres indiqués correspondent à l'ensemble des chimères qui pouvaient être considérées initialement comme appartenant à l'association (femelle antérieure) - mâle postérieure d'après l'aspect inhibé des gonades antérieures. Pour cette association ainsi que pour l'association $\sigma \leftrightarrow \sigma^{\circ}$, les nombres définitifs seront différents compte tenu de l'évolution des gonades antérieures à la suite de l'ablation des testicules postérieurs. 
- les chimères $(\wp) \leftrightarrow O^{*}$ ont toujours des testicules postérieurs bien développés; elles atteignent la maturité sexuelle mâle dans les mêmes délais que les chimères $\sigma^{*} \leftrightarrow \sigma^{*}$. La profonde inhibition des gonades antérieures n'autorise aucun diagnostic de leur sexe. La castration postérieure entraîne selon les cas au niveau antérieur : la présence d'ovaires, l'absence totale de gonades ou la présence de testicules;

- les chimères $O^{*} \leftrightarrow$ $\leftrightarrow$ ont le plus souvent 2 testicules antérieurs de même aspect ; les caractères externes de la maturité sexuelle mâle se manifestent tardivement ou même n'apparaissent pas. L'interprétation du tractus uro-génital de ces chimères doit toujours tenir compte de leur âge.

\section{1. - Chimères homosexuées $\sigma^{*} \leftrightarrow \sigma^{*}$}

19 chimères à corps double appartiennent à l'association mâle antérieuremâle postérieure. 15 animaux sont âgés au moins d'une année dont quatre ont dépassé 10 ans ; le plus âgé avait 11 ans et demi. Les caractères externes de la maturité sexuelle mâle sont apparus vers un an. On peut remarquer dès à présent, le nombre relativement faible de chimères $O^{*} \leftrightarrow O^{*}$ par rapport au nombre théorique attendu (19 animaux au lieu de 28). Dans la partie postérieure, les deux testicules sont toujours bien identifiables, par contre à l'avant, dans deux cas seulement les deux testicules sont bien développés; dans 17 autres cas, l'un des testicules est demeuré à l'état vestigial au voisinage d'un corps jaune adipeux (fig. 2). L'ablation des trois testicules bien développés fut pratiquée chez 3 chimères âgées de 31 mois. Trois à quatre mois après l'intervention, les caractères externes mâles avaient disparu, mais un an plus tard, ils étaient à nouveau apparents attestant ainsi l'évolution rapide du testicule vestigial en testicule normal à maturité sexuelle (PI. I, fig. 1). Les chimères $\sigma^{\circ} \leftrightarrow \sigma^{\text {* }}$ s'accouplent et émettent des spermatophores. S'il n'a pas encore pu être prouvé l'état fonctionnel simultané des testicules antérieurs et des testicules postérieurs chez le Pleurodèle, la preuve a été apportée pour les Axolotls $\sigma^{*} \leftrightarrow \sigma^{*}$ à corps double.

\section{2. - Chimères homosexuées $q \leftrightarrow$ q.}

26 chimères à corps double appartiennent à l'association femelle antérieurefemelle postérieure, nombre très proche de la prévision théorique (28). 23 animaux ont dépassé l'âge d'une année, la chimère la plus âgée a atteint 13 ans 2 mois. Les 2 ovaires postérieurs sont toujours présents. Pour les ovaires antérieurs, leur présence uni. ou bilatérale et leur importance par rapport aux ovaires postérieurs s'établissent comme suit :

- un seul ovaire antérieur, parfois plus volumineux que les deux ovaires postérieurs (6 cas) ;

- deux ovaires antérieurs plus petits que les ovaires postérieurs (9 cas) ;

- deux ovaires antérieurs peu différents des ovaires postérieurs (7 cas) (fig. 2) ;

- deux ovaires antérieurs plus volumineux que les ovaires postérieurs (4 cas). 


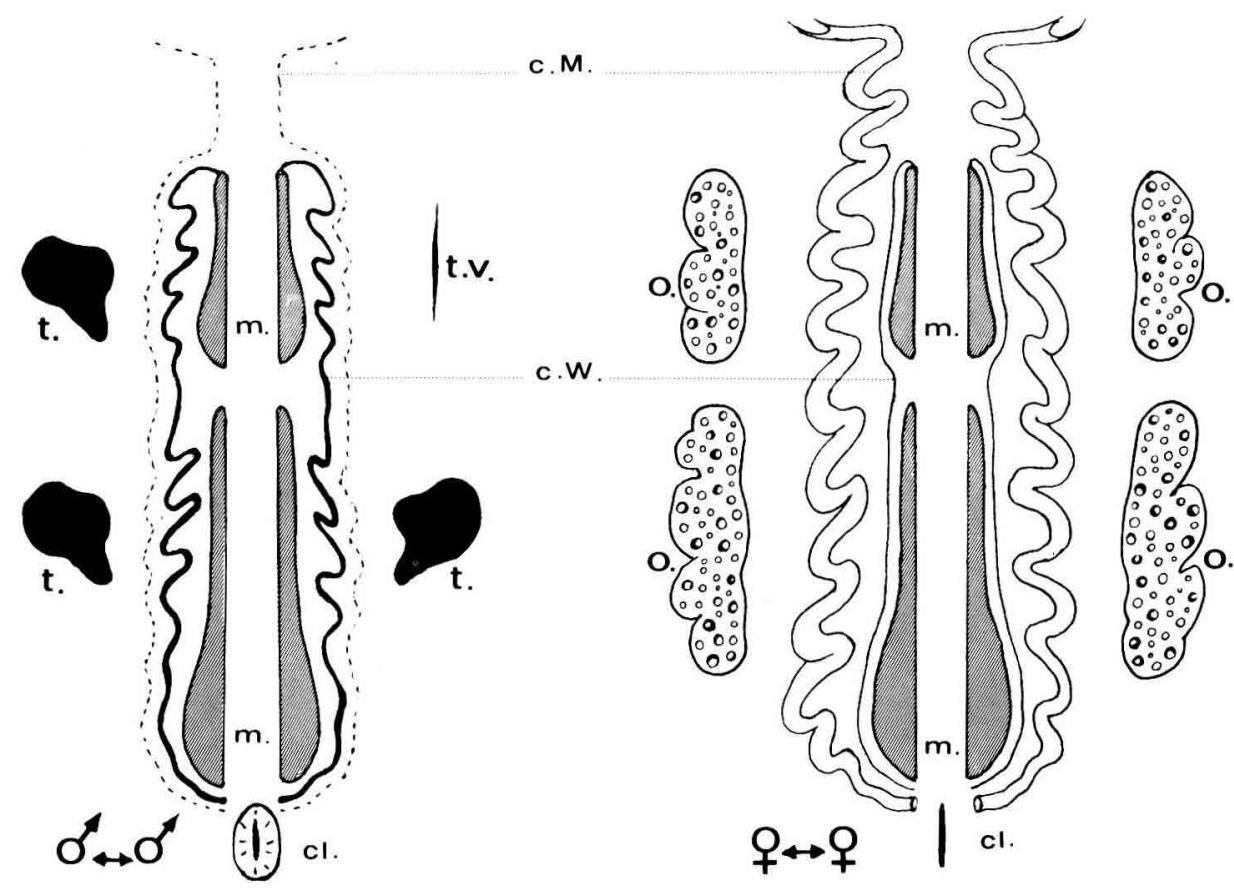

FIG. 2. - Représentation schématique du tractus uro-génital des chimères homosexuées à corps double adultes. $\sigma^{\prime} \leftrightarrow \sigma^{*}:$ chimère avec quatre testicules dont I'un est demeuré à l'état vestigial ; la continuité des canaux de Wolff permet l'émission simultanée des spermatozoïdes du testicule antérieur normal et des deux testicules postérieurs. $Q \leftrightarrow \leftrightarrow$ : chimère avec quatre ovaires ; la continuité des canaux de Müller permet l'émission simultanée des ovocytes des ovaires antérieurs et postérieurs.

(cl. : cloaque ; c.M. : canal de Müller ; c.W. : canal de Wolff ; m. : mésonéphros ; o. : ovaire ; o.i. : ovaire inhibé ; t. : testicule; t.v. : testicule vestigial).

Le début de la vitellogenèse qui va de pair avec la stimulation des canaux de Müller, intervient vers 12-14 mois. Quelle que soit leur position, les 3 ou 4 ovaires d'une chimère $\& \leftrightarrow q$ sont dans le même état d'évolution et atteignent la maturité sexuelle dans les mêmes délais que chez les femelles témoins. 3 chimères $\uparrow \leftrightarrow Q$ se sont reproduites à l'âge de 20 mois mais contrairement à ce qui a été

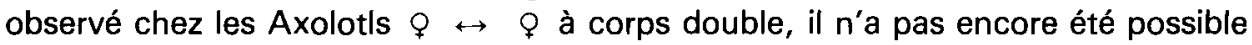
de reconnaître l'état fonctionnel simultané de tous les ovaires d'un Pleurodèle $९ \leftrightarrow$ \& à corps double.

\section{3. - Chimères hétérosexuées $(\emptyset) \leftrightarrow \circlearrowleft$.}

41 chimères à corps double pouvaient être considérées comme appartenant à l'association (femelle antérieure)-mâle postérieure ; elles sont toutes âgées d'au moins une année. Le début de la maturité sexuelle mâle qui peut s'observer dès 
cet âge, se manifeste le plus souvent à partir de 18-24 mois. Les 2 testicules postérieurs toujours présents ont le même aspect que chez les mâles témoins d'âge équivalent. Par contre, les gonades antérieures toujours extrêmement réduites, ont l'aspect d'une mince bandelette blanchâtre bordée, comme pour les gonades normales, d'un corps jaune adipeux qui permet de repérer la bandelette gonadique lors des laparotomies. II est exceptionnel de reconnaître macroscopiquement des ovocytes; dans 3 cas seulement et pour des animaux âgés d'un an, l'une des deux gonades antérieures présentait quelques ovocytes translucides. Une laparotomie exploratrice avait été pratiquée chez 6 animaux âgés d'un an ; au moment de la dissection 3 ou 4 années plus tard, les gonades antérieures furent retrouvées encore plus réduites, les ovocytes n'étaient plus discernables alors que les testicules postérieurs à maturité sexuelle avaient gardé leur aspect normal. Les canaux de Müller ne sont jamais stimulés, seuls les canaux de Wolff sont hypertrophiés comme chez les Pleurodèles mâles témoins à maturité sexuelle.

L'extrême réduction des gonades antérieures permettait, en première approximation, de considérer qu'il s'agissait d'ovaires inhibés sous l'influence des testicules postérieurs, ce qui correspondrait à l'effet free-martin classique. Même histologiquement, on n'observe que de rares cellules germinales ayant l'aspect de gonies primordiales, noyées dans du tissu conjonctif. Or cette structure se rencontre aussi bien pour les ovaires très inhibés qui se développent en présence de gonades mâles (Collenot et al., 1977) que pour les testicules demeurés vestigiaux en cas d'agénésie mésonéphrétique (Houillon, 1956). De plus, le nombre particulièrement élevé d'associations considérées $(Q) \leftrightarrow \sigma^{\prime}$ par rapport aux trois autres associations, est assez intrigant. Cependant, en absence de marqueur du sexe génétique, il n'est pas possible de préciser la nature exacte de ces gonades antérieures lors d'une laparotomie, sauf lorsque quelques ovocytes sont visibles. C'est pourquoi en vue de préciser le sexe et de suivre l'évolution des éléments gonadiques antérieurs, 22 chimères sur les 41 animaux considérés initialement comme $|\wp\rangle \leftrightarrow \circlearrowleft$, ont subi l'ablation des deux testicules postérieurs. Les 19 chimères non castrées postérieurement constituent le lot $A$ (fig. 3A).

La castration postérieure fut pratiquée à $10-11$ mois $(6$ cas), à 31 mois (14 cas) et à 44 mois ( 2 cas). Quel que soit leur âge au moment de l'intervention, toutes ces chimères présentaient les.caractères externes de la maturité sexuelle mâle ; ces caractères ont rapidement régressé et n'étaient plus discernables 4 à 5 mois après l'opération. Compte tenu de leur intérêt, les animaux ont fait l'objet de soins plus attentifs et furent élevés le plus longtemps possible. Seules les 2 chimères castrées à 44 mois furent sacrifiées 18 mois plus tard, toutes les autres ont péri ou furent sacrifiées au moins 32 mois plus tard et même pour certaines près de 10 ans après la castration. De la sorte, sur les 22 chimères ayant subi l'ablation des testicules postérieurs, 11 ont dépassé l'âge de 10 années ; la plus âgée est morte à 13 ans 2 mois.

$D^{\prime}$ après l'évolution de leurs gonades antérieures, les 22 chimères $(q) \leftrightarrow O^{\prime}$ castrées postérieurement se répartissent en 3 lots (fig. 3 ) :

- lot B : évolution ovarienne (3 cas), 

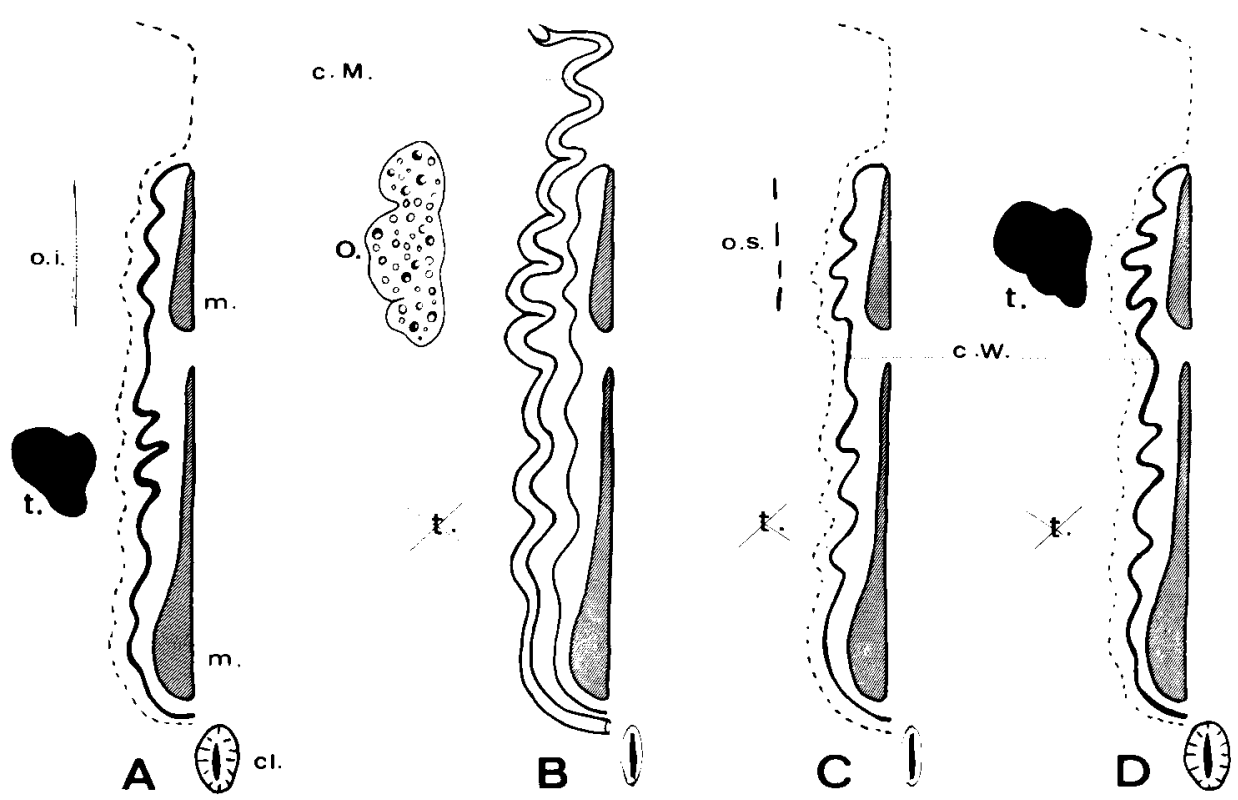

FIG. 3. - Représentation unilatérale schématique du tractus uro-génital des chimères hétérosexuées $Q \leftrightarrow \sigma$ à corps double adultes. A : chimère du lot $A$, ovaire antérieur inhibé et testicule postérieur d'aspect normal. $B, C$ et $D$ : mêmes chimères que $A$ mais après l'ablation des testicules postérieurs. B : évolution ovarienne. C : absence de gonade (o.s. : ovaire stérile). D : évolution testiculaire.

Même légende que pour la figure 2 .

\section{PLANCHE I}

Pleurodèles à corps double chimères hétérosexuées $Q \leftrightarrow \sigma^{\prime}$ (sauf figure 1). Toutes les chimères ont subi l'ablation des deux testicules postérieurs à l'âge de 31 mois. Les gonades castrées, fixées et conservées, sont photographiées en même temps que le tractus uro-génital tel qu'il a évolué plusieurs années après la castration. Les gonades castrées placées sur un fond noir avec leur corps jaune adipeux, correspondent rigoureusement à chaque animal.

FIG. 1. - Chimères homosexuées $O * \leftrightarrow \sigma(10$ ans, $205 \mathrm{~mm})$, développement d'un testicule qui était resté vestigial après I'ablation des trois testicules normaux pratiquée 7 ans et demi auparavant.

FIG. 2. - Chimère du lot $B(78$ mois, $210 \mathrm{~mm})$. Les deux ovaires antérieurs inhibés ont évolué en ovaires à maturité sexuelle après l'ablation des deux testicules postérieurs pratiquée 47 mois auparavant.

FIG. 3. - Chimère du lot C $(13$ ans 2 mois, $220 \mathrm{~mm})$. Les deux testicules postérieurs et l'ovaire antérieur inhibé situé du côté gauche ont été enlevés 10 ans et demi auparavant. L'inhibition ovarienne totale (ovaire stérile) se traduit par la seule présence du corps jaure adipeux du côté droit.

FIG. 4. - Chimère du lot D (10 ans et demi, $220 \mathrm{~mm})$. Les deux testicules postérieurs et l'ovaire antérieur situé du côté gauche ont été enlevés 8 ans auparavant. La gonade antérieure située du côté droit qui avait le même aspect que l'ovaire inhibé gauche au moment de la castration, a évolué en testicule à maturité sexuelle.

c.j. : corps jaune adipeux ; c.M. : canal de Müller ; c.W. : canal de Wolff ; m. : mésonéphros ; o. : ovaire; o.i. : ovaire inhibé ; t. : testicule. Toutes les figures sont à la même échelle. 

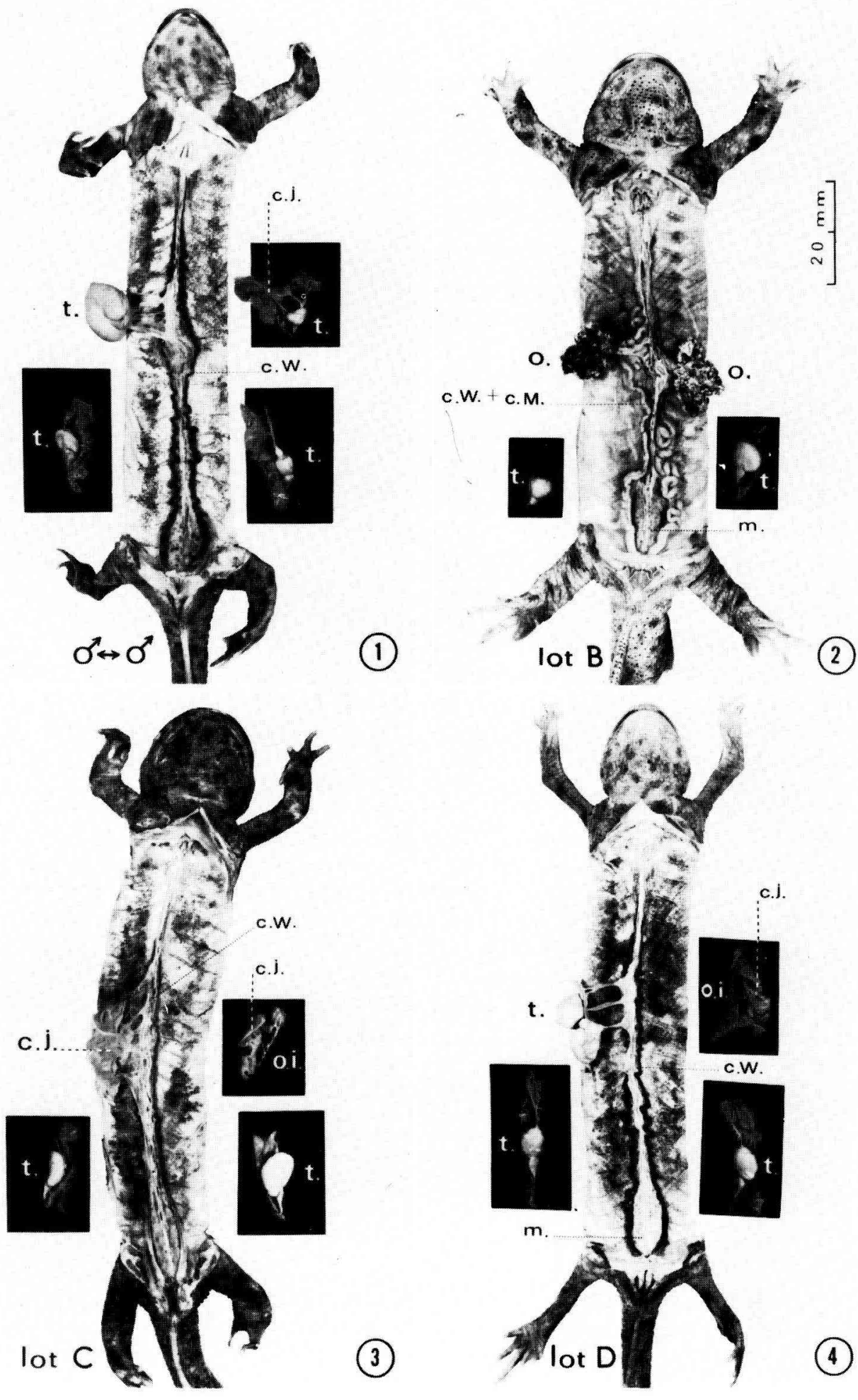

(1)

c.W. + c.M.
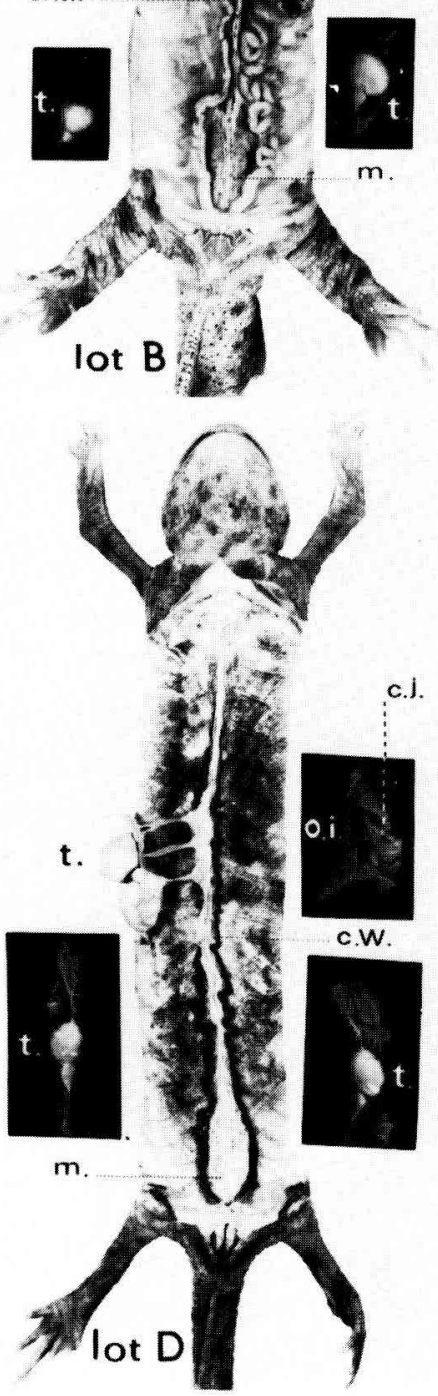
- lot C : absence de gonades (4 cas),

- lot D : évolution testiculaire (15 cas).

Chimères du lot $B$ : évolution ovarienne. - Les 3 chimères dont les gonades antérieures ont évolué en ovaires avaient subi l'ablation des testicules postérieurs à 31 mois. Chez l'une d'elles, un ovaire à maturité sexuelle fut déjà reconnu lors d'une laparotomie $\mathbf{2 4}$ mois après la castration ; elle fut sacrifiée à l'âge de 64 mois (Houillon, 1977, fig. 7).

Les 2 autres chimères dont les minces cordons gonadiques antérieurs étaient encore dans le même état 27 mois après la castration postérieure, furent sacrifiées respectivement à l'âge de 78 mois et de 130 mois, soit 47 et 99 mois après la castration. Elles présentaient alors des ovaires antérieurs à maturité sexuelle (PI. I, fig. 2). L'évolution ovarienne est intervenue très tardivement dans ces 2 cas. A noter chez les 3 chimères que la stimulation des canaux de Müller est allée de pair avec le développement ovarien. Cependant les oviductes ne sont jamais aussi hypertrophiés que chez les femelles témoins, de la sorte et malgré leur maturité, les ovocytes n'auraient jamais pu être pondus.

Chimères du lot $C$ : absence de gonades. -4 chimères ayant subi l'ablation des testicules postérieurs à 10 mois pour l'une et à 31 mois pour les 3 autres, n'ont présenté par la suite aucun développement de leurs gonades antérieures. Deux d'entre elles furent sacrifiées 3 ans après la castration et les deux autres respectivement 9 ans et demi et 10 ans et demi après la castration. Leurs gonades antérieures sont demeurées dans le même état ou dans un état encore plus inhibé que lors de la castration (PI. I, fig. 3). Les canaux de Wolff restent bien marqués et attestent la maturité sexuelle mâle au moment de l'ablation des testicules postérieurs.

Chimères du lot $D$ : évolution testiculaire. - 15 chimères dont les gonades antérieures ont évolué en testicules avaient été castrées postérieurement à 1011 mois ( 4 cas), à 31 mois ( 9 cas) et à 44 mois ( 2 cas). II fut inutile de pratiquer par la suite une laparotomie pour suivre l'évolution gonadique puisque la réapparition des caractères sexuels externes mâles suffisait pour révéler à nouveau la présence de testicules à maturité. Pour 8 chimères les caractères étaient déjà bien évidents 12 mois après la castration, en particulier pour les 2 animaux castrés à 44 mois; pour les 7 autres chimères, les caractères de la maturité sexuelle mâle sont réapparus 20 à 24 mois après la castration. Dans ce lot, 8 chimères ont dépassé l'âge avancé de 10 années. Lorsque seulement l'une des deux gonades antérieures évolue en testicule, ce dernier est souvent très volumineux (PI. I, fig. 4).

\section{4. - Chimères hétérosexuées Ơ $^{*}$ Q .}

L'analyse porte sur 29 chimères à corps double appartenant à l'association mâle antérieure-femelle postérieure ; 26 d'entre elles ont atteint ou dépassé l'âge d'une année. Les caractères externes de la maturité sexuelle mâle qui ne se mani- 
festent pas dans tous les cas, ont quelquefois été reconnus à un an et le plus souvent vers 2 ans et même plus tard. 3 chimères ont atteint l'âge de 10 ans, l'une d'elles fut sacrifiée à 15 ans. Il s'agissait en l'occurrence de la longévité maximum rencontrée chez les Pleurodèles chimères à corps double.

Les testicules antérieurs sont toujours reconnus facilement à l'observation macroscopique. Dans la moitié des cas, les 2 testicules ont le même aspect, dans les autres, l'une des 2 gonades est plus ou moins réduite et même dans 5 cas, l'un des testicules antérieurs est resté vestigial. Un dimorphisme comparable des testicules antérieurs se rencontre aussi, et même plus fréquemment, chez les chimères homosexuées $\sigma^{*} \leftrightarrow$ ơ (fig. 2).

Les gonades postérieures des chimères hétérosexuées $\sigma^{\prime} \leftrightarrow$ à corps double offrent une très grande diversité d'aspect depuis l'inhibition ovarienne subtotale (effet free-martin) jusqu'au développement pratiquement complet d'ovaires à maturité sexuelle (très peu ou pas d'effet free-martin). Chaque animal constitue un cas particulier. Néanmoins, d'après l'aspect des gonades postérieures observées lors des laparotomies et leur évolution, les 29 chimères $\sigma^{\prime} \leftrightarrow$ peuvent être réparties en quatre lots (fig. 4):

- lot E : inhibition ovarienne précoce $(11$ cas),

- lot $F$ : inhibition ovarienne tardive (6 cas),

- lot $\mathrm{G}$ : inhibition réciproque ou équilibre (10 cas),

- lot $\mathrm{H}$ : inhibition testiculaire tardive (2 cas).

Chimères du lot $E$ : inhibition ovarienne précoce. - Ce phénomène, qui correspond à l'effet free-martin, est intervenu chez 11 animaux. Les ovaires postérieurs sont réduits à l'état de simples cordons blanchâtres dès l'âge de 5 mois ; aucun ovocyte n'est discernable quel que soit l'âge (à l'exception des 3 chimères castrées antérieurement analysées ci-après). Les gonades postérieures ont le même aspect inhibé que les gonades antérieures des chimères $(O) \leftrightarrow \sigma$ du lot $A$. Ce tractus gonadique reconnu à la laparotomie pour 3 chimères âgées d'un an, fut retrouvé dans le même état 5 à 6 ans plus tard.

Trois autres chimères de ce lot ont subi l'ablation des testicules antérieurs ; observées au moment de l'intervention, leurs gonades postérieures étaient profondément inhibées. Une première chimère castrée antérieurement à 31 mois a subi une nouvelle laparotomie exploratrice 27 mois plus tard. Son tractus gonadique ne semblait pas s'être modifié, et pourtant, lorsqu'elle fut sacrifiée à l'âge de 8 ans, soit plus de 5 ans après la castration antérieure, elle avait 2 ovaires postérieurs à maturité sexuelle (PI. II, fig. 7).

Une deuxième chimère, également castrée antérieurement à 31 mois, a subi aussi une laparotomie exploratrice 27 mois plus tard, mais cette fois un ovaire s'était déjà développé postérieurement avec des ovocytes en fin de vitellogenèse. La présence d'un seul ovaire tient à ce que le tractus gonadique situé de l'autre côté avait été enlevé en même temps que les testicules antérieurs en vue de l'étude histologique. Cette chimère fut sacrifiée à l'âge de 15 ans, soit plus de 12 années après la castration antérieure. Malgré l'atrésie ovocytaire due à l'âge avancé, I'ovaire renfermait encore de nombreux ovocytes mûrs (PI. II, fig. 5). 

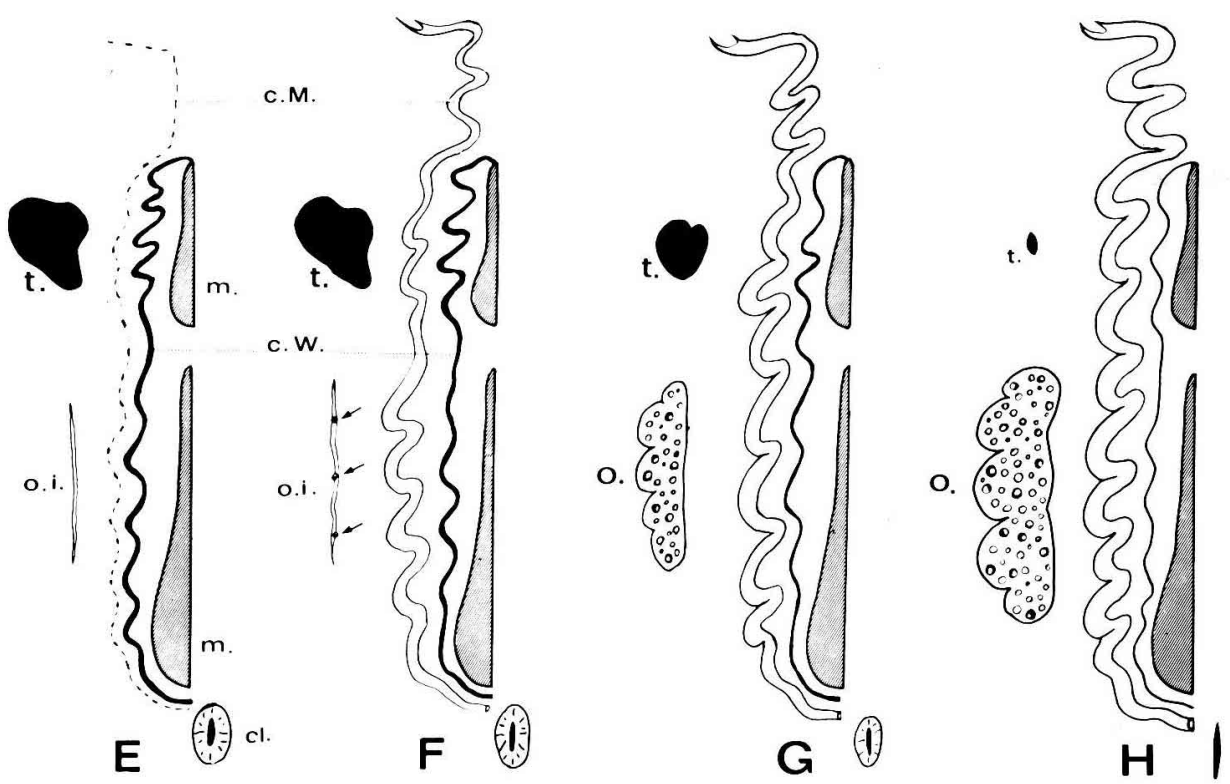

FIG. 4. - Représentation unilatérale schématique du tractus uro-génital des chimères hétérosexuées $\sigma^{\circ} \leftrightarrow$ Q à corps double adultes. $\mathrm{E}:$ chimère du lot $\mathrm{E}$, inhibition ovarienne précoce, testicule antérieur d'aspect normal et ovaire postérieur inhibé. $F$ : chimère du lot $F$, inhibition ovarienne tardive, persistance de petits ovocytes (flèches) et stimulation du canal de Müller. G : chimère du lot $G$, inhibition réciproque ou équilibre entre le testicule antérieur et l'ovaire postérieur (animal hermaphrodite). $\mathrm{H}$ : chimère du lot $\mathrm{H}$, inhibition testiculaire tardive, persistance d'une légère hypertrophie des canaux de Wolff.

Même légende que pour la figure 2 .

\section{PLANCHE II}

Pleurodèles à corps double chimères hétérosexuées $\sigma^{*} \leftrightarrow \quad$. Les gonades castrées ont été placées sur un fond noir pour la prise de vue du tractus uro-génital tel qu'il a évolué plusieurs années après la castration.

FIG. 5. - Chimère du lot E (15 ans, $215 \mathrm{~mm}$ ) ayant subi l'ablation des 2 testicules antérieurs et de l'ovaire inhibé postérieur gauche à l'âge de 31 mois. La gonade postérieure située du côté droit qui avait le même aspect que l'ovaire inhibé gauche, a évolué en ovaire à maturité sexuelle.

FIG. 6. - Chimère du lot E (12 ans 2 mois, $245 \mathrm{~mm})$ ayant subi l'ablation du testicule normal et du testicule vestigial (t.v.) antérieurs à l'âge de 10 mois. Postérieurement, les 2 ovaires étaient inhibés, celui de droite a évolué en ovaire avec des ovocytes mûrs et celui de gauche a subi une inversion de son phénotype sexuel pour évoluer en testicule.

FIG. 7. - Chimère du lot $E(8$ ans, $215 \mathrm{~mm})$ ayant subi l'ablation des 2 testicules antérieurs à l'âge de 31 mois. Les 2 ovaires postérieurs qui étaient encore inhibés 27 mois après la castration, ont évolué tardivement en ovaires à maturité sexuelle. Noter pour les figures 2, 5, 6 et 7 la faible stimulation des canaux de Müller malgré la présence d'ovaires à maturité sexuelle.

FIG. 8. - Chimère du lot $F(4$ ans 2 mois, $225 \mathrm{~mm}$ ). L'inhibition ovarienne tardive est attestée par la présence d'ovocytes en cours d'atrésie (flèche noire) et la stimulation des canaux de Müller. Noter l'abouchement du canal de Müller antérieur droit dans le canal de Müller gauche (flèche en tirets).

FIG. 9. - Chimère du lot $G(3$ ans, $210 \mathrm{~mm})$. Testicules antérieurs et ovaires postérieurs à maturité sexuelle (animal hermaphrodite). Pour les figures 8 et 9 malgré la présence de testicules à maturité sexuelle, les canaux de Müller sont aussi stimulés que chez une femelle normale. Même légende que pour la Planche I. Toutes les figures sont à la même échelle. 


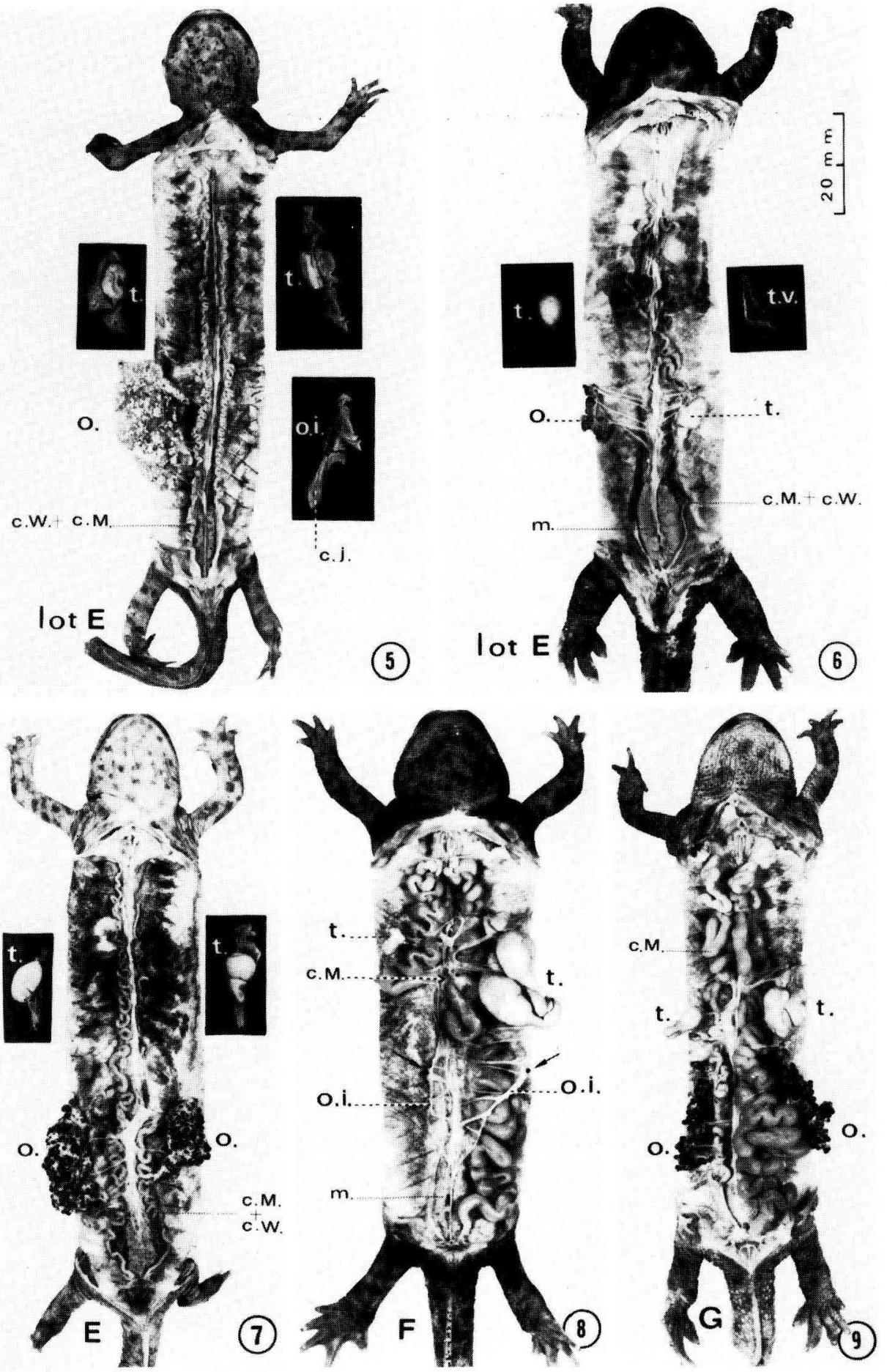


Une troisième chimère fut castrée antérieurement à 10 mois ; elle a péri à l'âge de 12 ans 2 mois (soit plus de 11 années après). Si l'une de ses gonades postérieures a encore bien évolué en ovaire, de taille réduite mais avec des ovocytes mûrs, par contre l'autre gonade postérieure a évolué en testicule (PI. II, fig. 6). Une telle situation est unique à travers les 115 chimères à corps double analysées.

Les 3 chimères ci-dessus du lot $E$ présentaient les caractères externes de la maturité sexuelle mâle au moment de l'ablation des testicules antérieurs et leurs canaux de Wolff étaient bien hypertrophiés. Les caractères externes se sont atténués progressivement après la castration mais les canaux de Wolff sont encore marqués au moment de la dissection bien qu'en partie masqués par le développement tardif des canaux de Müller consécutif à l'évolution ovarienne des gonades postérieures. Cependant les oviductes ne sont jamais aussi stimulés que chez les femelles témoins à maturité sexuelle, de sorte que ces chimères n'auraient certainement jamais pu émettre leurs ovocytes tout comme les chimères $q \leftrightarrow$ ơ dont les ovaires antérieurs ont évolué très tardivement, après l'ablation des testicules postérieurs.

Chimères du lot $F$ : inhibition ovarienne tardive. - Ce phénomène a été reconnu chez 6 chimères qui ont subi une laparotomie exploratrice vers l'âge d'un an. Les testicules antérieurs sont plus ou moins développés, les caractères externes de la maturité sexuelle mâle débutaient chez un animal. Les ovaires postérieurs bien nets renferment de nombreux ovocytes translucides et même quelques uns amorcent la vitellogenèse. Le début de la vitellogenèse coïncidant toujours avec le début de la stimulation des canaux de Müller, toutes les chimères ont des oviductes bien marqués. Mais le développement ovarien ne semble pas pouvoir se poursuivre plus avant, au contraire, les ovaires régressent vers l'âge de 2 ans alors que les caractères externes mâles deviennent plus évidents. La régression ovarienne devient telle qu'à 4 ans il ne reste qu'un mince tractus gonadique postérieur avec parfois encore quelques petits ovocytes translucides. Les glandes génitales des chimères du lot $F$ finissent par présenter, mais très tardivement, le même aspect que dans le lot $E$ lorsque les testicules antérieurs demeurent en place. La seule différence anatomique porte sur les canaux de Müller dont la stimulation rappelle la présence d'ovaires proches de la maturité sexuelle quelques années auparavant (PI. II, fig. 8).

Chimères du lot $G$ : inhibition réciproque ou équilibre. - Ce lot comprend 10 chimères dont les gonades antérieures et postérieures ont la même importance (petites, moyennes ou volumineuses) et paraissent se trouver dans le même état d'évolution (juvéniles, proches de la maturité sexuelle ou à maturité sexuelle). Chez les 3 plus jeunes, âgées de 5 mois, l'aspect des testicules antérieurs et celui des ovaires postérieurs rappellent, à la taille près, l'aspect des gonades chez les animaux témoins au même âge. Deux chimères âgées respectivement de 12 et de 31 mois, ont des gonades peu développées mais réduites dans les mêmes proportions; il semble s'être produit une inhibition réciproque entre les gonades antérieures et les gonades postérieures.

Le classement des chimères dans le lot $G$ repose surtout sur les observations à partir des laparotomies chez 4 animaux à l'âge de 11 mois. Pour 2 d'entre eux, 
les ovaires postérieurs étaient encore à l'état juvénile bien que renfermant de nombreux ovocytes translucides; pour les 2 autres, la vitellogenèse débutait. Un an plus tard, les 4 chimères présentaient une légère turgescence du cloaque annonçant la maturité sexuelle mâle. La dissection pratiquée à cet âge pour 3 animaux et un an après pour le quatrième, a révélé la présence à la fois de testicules antérieurs à maturité sexuelle et d'ovaires postérieurs avec de nombreux ovocytes en fin de vitellogenèse (PI. II, fig. 9). Ces 4 chimères ainsi qu'une cinquième, peuvent être considérées comme de véritables animaux hermaphrodites capables de produire en même temps des spermatozoïdes et des ovocytes mûrs.

Chimères du lot $H$ : inhibition testiculaire tardive. -2 chimères reconnues comme $\sigma^{\prime} \leftrightarrow$ ᄋ à la suite d'une laparotomie, ont eu une évolution particulière. Pour l'une, la laparotomie à 8 mois avait révélé des testicules antérieurs bien nets et des ovaires postérieurs également bien développés avec un début de vitellogenèse, ce qui est assez rare pour cet âge surtout chez les:animaux chimères. Elle fut sacrifiéé à l'âge de deux ans, soit 16 mois plus tard. Les 2 testicules antérieurs n'avaient pas évolué, par contre les ovaires postérieurs s'étaient bien développés et renfermaient de nombreux ovocytes mûrs. Pour la seconde chimère, la laparotomie à 8 mois avait révélé des nodules testiculaires antérieurs et des ovaires postérieurs plus importants que chez la chimère précédente avec également un début de vitellogenèse. Cet animal fut sacrifié à 4 ans 2 mois, soit plus de 3 ans et demi après la laparotomie. Les testicules antérieurs $n$ 'ont pas été retrouvés alors que les ovaires postérieurs étaient comparables à ceux d'une femelle témoin à maturité sexuelle. Par conséquent ici, les testicules ont totalement régressé au bénéfice des ovaires. II est vraisemblable que la chimère précédente, compte tenu de son aspect à 8 mois et de l'absence d'évolution des testicules 16 mois plus tard, aurait manifesté le même phénomène d'inhibition testiculaire si elle n'avait pas été sacrifiée à 24 mois.

\section{Discussion.}

L'une des originalités du présent travail est de s'adresser à un grand nombre d'animaux, ce qui permet de mettre l'accent sur des phénomènes qui pouvaient paraître exceptionnels auparavant. Egalement, l'élevage des animaux expérimentaux le plus longtemps possible, pratique désormais peu courante, autorise des conclusions nouvelles et montre à quel point leur physiologie n'est pas perturbée en dépit d'un corps double. II s'établit une régulation anatomique aux stades embryonnaires suivie d'une régulation physiologique telle, que les chimères sont en tous points comparables, à la morphologie près, aux animaux témoins. La tolérance immunologique à propos de ces chimères allogéniques à corps double mériterait une attention encore plus particulière que pour les chimères à corps simple (Goujon, 1974).

Une autre originalité est la pratique systématique des laparotomies exploratrices accompagnées souvent de l'ablation des testicules qui ont permis de révéler tous les degrés d'interactions entre glandes génitales de sexe différent depuis 
l'inhibition à peine marquée des ovaires jusqu'à leur inversion complète. Pour les chimères homosexuées $\sigma^{*} \leftrightarrow O^{*}$ et $\wp_{\leftrightarrow}$ Q ainsi que pour les chimères hétérosexuées $(\wp) \leftrightarrow$ ơ sans castration postérieure, les observations au cours des laparotomies furent toujours confirmées plus tard au moment de la dissection. Mais pour les chimères hétérosexuées $O^{\prime} \leftrightarrow$ Q, l'aspect des gonades observées à 1,2 ou 3 ans n'implique nullement un aspect comparable quelques années après. L'interprétation du phénomène ultime comme l'inhibition ovarienne tardive ou l'inhibition testiculaire ne reposent plus sur l'extrapolation spéculative d'états antérieurs dès lors que pour chaque animal, ces états antérieurs ont été observés avec précision.

Les chimères homosexuées atteignent la maturité sexuelle dans les mêmes délais que les animaux témoins. Elles s'accouplent, les chimères $q \leftrightarrow q$ ont émis des œufs. Ceci implique une parfaite continuité de l'avant vers l'arrière, des canaux de Wolff chez les mâles et des canaux de Müller chez les femelles. Cette continuité est effective dans la majorité des cas. On observe toutefois chez certaines chimères $\subsetneq \leftrightarrow q$ une absence unilatérale et en arrière d'un canal de Müller. Du même côté et en avant, le canal de Müller est bloqué au niveau de la soudure entre les parties antérieure et postérieure de la chimère ; il se termine le plus souvent par une extrémité aveugle, très dilatée lorsqu'elle renferme des ovocytes qui n'ont pas pu être évacués. L'extrémité distale d'un canal de Müller antérieur peut parfois se raccorder au canal de Müller situé de l'autre côté ; le tractus müllérien dans ce cas affecte la forme de la lettre $Y$ avec deux canaux antérieurs pourvus de leur ostium et un unique canal postérieur (PI. II, fig. 8).

La présence de deux gonades postérieures normales est la règle chez toutes les chimères homosexuées. La réduction de l'une des deux gonades antérieures relève soit d'une déficience du composant somatique en relation avec une agénésie du mésonéphros (Houillon, 1956), soit d'une déficience du composant germinal (Dournon, 1977). L'origine de ces deux déficiences, parfois associées, provient de la technique opératoire qui ne peut éviter une perte de matériel gonadique organo-formateur au moment de la section et lors des manipulations pour raccorder les deux hémi-parties d'une future chimère. D'ailleurs, la réduction de l'une des deux gonades se rencontre aussi chez certaines chimères à corps simple obtenues selon la même technique microchirurgicale.

Pour les gonades antérieures, un autre phénomène semble intervenir notamment à propos de la réduction de l'un des testicules (testicule vestigial). Pour les chimères homosexuées $\sigma^{\prime} \leftrightarrow{ }^{\circ}$, seulement 2 animaux sur 19 avaient les 2 testicules antérieurs bien développés alors que pour les chimères hétérosexuées ơ $\leftrightarrow$ १ chez 14 animaux sur 29, les 2 testicules antérieurs sont bien développés. II apparaît clairement que le développement des testicules antérieurs est bien plus souvent perturbé par le développement de testicules postérieurs normaux que par le développement d'ovaires postérieurs plus ou moins réduits.

La greffe embryonnaire en chimère à corps double est avant tout l'occasion de réaliser des associations hétérosexuées dans $50 \%$ des cas. L'analyse du phénomène de free-martin chez les Amphibiens, à savoir l'inhibition du développement ovarien en présence d'un développement testiculaire comme chez les Bovidés (Jost et al., 1975), revêt alors un nouvel intérêt. Le résultat original et essen- 
tiel qui se dégage désormais chez les Amphibiens est qu'il convient de prendre en compte l'effet de position dans les interactions entre glandes génitales de sexe opposé : l'inhibition ovarienne se manifeste avec des degrés très divers selon la position antérieure ou postérieure des ovaires par rapport aux testicules.

Dans les chimères hétérosexuées considérées $(\emptyset) \leftrightarrow \sigma^{*}$, les gonades antérieures sont toujours extrêmement réduites et demeurent telles tant que les testicules postérieurs sont en place et celà, indépendamment de l'âge des animaux (lot A). Mais la castration postérieure va modifier cet état. Pour les chimères du lot $B$ ( 3 cas) les gonades antérieures qui évoluent en ovaires, sont effectivement des gonades femelles, certes très inhibées, mais non stériles. La castration postérieure levant l'inhibition, l'évolution ovarienne peut alors intervenir, parfois après un délai de plusieurs années. Les chimères du lot $\mathrm{B}$ correspondent bien à des associations hétérosexuées ovaires antérieurs - testicules postérieurs. Pour les animaux du lot $C$ ( 4 cas), les gonades antérieures sont des ovaires stériles qui ne peuvent pas évoluer, même après de nombreuses années, par suite d'une inhibition ovarienne totale. Pour les animaux du lot $D(15 \mathrm{cas})$, les gonades antérieures qui évoluent en testicules sont pour les unes des gonades mâles demeurées longtemps vestigiales et pour les autres, des gonades femelles ayant subi une inversion de leur phénotype sexuel comme le montrent les résultats ci-après. Sur un total de 22 chimères considérées $(\wp) \leftrightarrow \sigma^{\prime}$ castrées postérieurement, 15 animaux $(68 \%)$ ont différencié ultérieurement des testicules antérieurs. On peut donc admettre que sur les 41 chimères considérées initialement $(\varphi) \leftrightarrow \sigma^{\circ}, 28$ auraient eu une évolution testiculaire antérieure après castration postérieure. Les 13 autres chimères auraient de toute évidence, présenté antérieurement soit une évolution ovarienne soit une absence de gonades.

Ainsi sur les 41 chimères initialement considérées comme $(q) \leftrightarrow 0^{\prime}, 28$ se classent désormais parmi les chimères $\sigma^{*} \leftrightarrow O^{\prime}$ et 13 parmi les chimères $\leftrightarrow \sigma^{*}$. Pour l'ensemble des 115 chimères, l'effectif corrigé correspondant aux 4 combinaisons sexuelles est alors le suivant :

- chimères $O^{*} \leftrightarrow O^{*}: 19+28=47$ cas $(41 \%)$,

- chimères $\uparrow \leftrightarrow$ ९ : 26 cas $(23 \%)$,

- chimères $\& \leftrightarrow \sigma^{\circ}: \quad 13$ cas $(11 \%)$,

- chimères $\sigma^{*} \leftrightarrow$ \&: $\quad 29$ cas $(25 \%)$.

L'élevage du Pleurodèle pratiqué dans les conditions habituelles conduit toujours à une sex-ratio normale (Dournon et Houillon, 1984). Par conséquent, chacune des combinaisons sexuelles doit théoriquement comprendre 28 animaux. Le nombre de chimères $\% \leftrightarrow q(26)$ et le nombre de chimères $\sigma^{*} \leftrightarrow q$ (29) sont remarquablement proches du nombre théorique (28). De plus, le nombre total de chimères $\sigma^{\circ} \leftrightarrow \sigma^{*}$ et de chimères $\odot \leftrightarrow \sigma^{*}$ (60 animaux) est également très proche du nombre total théorique pour ces deux combinaisons (57). La concordance entre les nombres réels et les nombres théoriques non seulement atteste l'existence d'une sex-ratio normale dans les populations de Pleurodèles utilisées pour obtenir les 115 chimères à corps double mais donne en outre une signification toute particulière au nombre important de chimères $O^{*} \leftrightarrow O^{*}$ (47 au lieu de 28) et au faible nombre de chimères $q \leftrightarrow O^{*}(13$ au lieu de 28$)$. 
Les 47 chimères O $^{*} \leftrightarrow \sigma^{*}$ correspondent aux 19 chimères $\sigma^{*} \leftrightarrow \sigma^{*}$ initialement reconnues, à une dizaine de chimères $\sigma^{*} \leftrightarrow \sigma^{\prime}$ qui manquent dans cette combinaison pour atteindre le nombre théorique de 28 animaux et à une quinzaine de chimères $(q) \leftrightarrow O^{*}$ dont les gonades antérieures ont évolué en testicules.

La dizaine de chimères $O^{*} \leftrightarrow$ O manquantes avait été prise en compte initialement comme $(Q) \leftrightarrow \sigma^{\prime}$ alors que les deux gonades antérieures étaient en réalité des testicules vestigiaux qui ont évolué en testicules normaux après l'ablation des testicules postérieurs. On retrouve ici, encore plus marqué, le phénomène énigmatique déjà observé : des testicules postérieurs inhibent non seulement l'un mais parfois les deux testicules antérieurs.

Aux 13 chimères $Q \leftrightarrow \circlearrowleft$, il manque une quinzaine d'individus pour atteindre le nombre théorique. Ces chimères manquantes avaient bien été initialement prises en compte comme $(\wp) \leftrightarrow{ }^{\prime}$, leur partie antérieure provenait bien d'un embryon génétiquement femelle, cependant la différenciation ovarienne fut non seulement inhibée mais inversée pour conduire à une différenciation testiculaire. Les gonades antérieures inversées demeurent à l'état vestigial tant que les testicules postérieurs sont présents, elles ont évolué en testicules d'apparence normale après la castration postérieure.

L'interprétation ci-dessus confirme les résultats obtenus par Collenot et al. (1977) chez quelques chimères $९ \leftrightarrow$ o dont le génotype sexuel était connu avec certitude au moment de l'intervention embryonnaire : 5 de ces animaux furent castrés postérieurement, pour 4 d'entre eux la castration postérieure étant incomplète, les gonades antérieures sont demeurées à l'état vestigial. Par contre pour le cinquième, l'absence définitive de testicules postérieurs a permis l'évolution testiculaire d'une gonade antérieure à génotype sexuel femelle.

L'évolution testiculaire tardive des gonades antérieures après la castration postérieure chez les chimères $\bigcirc \leftrightarrow \sigma^{\prime}$ rappelle une fois de plus l'action inhibitrice des testicules postérieurs sur les gonades de même nature situées en avant. Mais surtout le fait de n'obtenir que 13 chimères $\subsetneq \leftrightarrow O^{*}$ au lieu d'au moins le double d'après la prévision théorique, prouve que l'inversion du phénotype sexuel gonadique dans les associations hétérosexuées $\uparrow \leftrightarrow \circlearrowleft$ est un phénomène assez fréquent puisque d'après les considérations ci-dessus, il intervient au moins dans la moitié des cas.

Les chimères hétérosexuéès $\sigma^{\prime} \leftrightarrow$ Q sont loin de présenter un tractus urogénital comparable, à la disposition des gonades près, au tractus uro-génital des chimères hétérosexuées $\wp \leftrightarrow \sigma^{*}$. Les gonades mâles, qu'elles soient antérieures ou postérieures, ont le plus souvent un développement normal, mais alors que les ovaires antérieurs sont toujours très inhibés (lot $A$ ), une inhibition du même ordre ne se rencontre pour les ovaires postérieurs que seulement dans 11 cas sur 29 animaux (lot E). Dans tous les autres cas, les ovaires postérieurs sont bien discernables du moins jusqu'à l'âge d'un an, avec des ovocytes en vitellogenèse. Si l'inhibition ovarienne se produit, elle se manifeste beaucoup plus tardivement (lot F).

L'évolution des gonades chez les chimères des lots $E$ et $F$ rappelle l'évolution des gonades intersexuées obtenues après traitement par l'hormone femelle (Gallien, 1962). Ces dernières présentent le plus souvent un nodule testiculaire antérieur et des éléments ovariens postérieurs, disposition identique à celle des gona- 
des chez les chimères $\sigma^{*} \leftrightarrow$ \&. Au même titre, la " conversion mâle \#, rapide ou différée, des gonades intersexuées est comparable dans son résultat à l'inhibition ovarienne, précoce ou tardive, chez les chimères $\sigma \leftrightarrow \leftrightarrow q$ des lots $E$ et $F$. Mais alors qu'il suffit d'un nodule testiculaire pour qu'une gonade intersexuée évolue toujours en testicule, il en va tout autrement pour les gonades des chimères hétérosexuées des lots $G$ et $H$. Chez les animaux du lot $G$, il s'instaure très tôt une inhibition mutuelle ou une sorte d'équilibre permanent entre les testicules antérieurs et les ovaires postérieurs. Rencontré dès l'âge de 5 mois, cet état était encore évident et sans modification 2 à 3 ans plus tard; il aboutit aux chimères hermaphrodites. Ces dernières seraient toujours demeurées telles, sans manifester d'inhibition ovarienne tardive. En effet, chez les chimères du lot $F$ qui ont présenté une inhibition ovarienne tardive, ce phénomène était déjà évident vers l'âge de 2 ans et demi.

Pour les 2 chimères du lot $\mathrm{H}$, il n'y a même plus d'équilibre comme dans le lot $\mathrm{G}$, on assiste à une véritable inhibition testiculaire tardive. Bien qu'ayant présenté à un certain moment des testicules antérieurs, l'évolution gonadique ultime se fait au seul bénéfice des ovaires postérieurs. Limitée à 2 cas sur 29 chimères $\sigma \leftrightarrow Q$, cette évolution originale déjà signalée à propos de la parabiose chez les Urodèles (Burns, 1935), n'a pu être reconnue que grâce aux laparotomies exploratrices précoces.

Compte tenu de l'action inhibitrice relativement peu importante des testicules antérieurs sur les ovaires postérieurs, on pouvait prévoir que l'inversion du phénotype ovarien interviendrait peu souvent. D'ailleurs le nombre de chimères o $\leftrightarrow$ \& (29 animaux), remarquablement conforme au nombre théorique attendu, est déjà une première indication. De plus, même si seulement 3 chimères sur 11 du lot $E$ furent castrées antérieurement, dans les 3 cas, des ovaires postérieurs se sont développés. Parmi ceux-ci se place tout de même l'animal unique qui a présenté postérieurement un ovaire d'un côté et un testicule de l'autre. Contrairement aux chimères hétérosexuées $\wp \leftrightarrow \sigma^{*}$ chez lesquelles l'inversion des ovaires antérieurs est assez fréquente, chez les chimères hétérosexuées $0^{*} \leftrightarrow q$ l'inversion des ovaires postérieurs est tout à fait exceptionnelle.

Les chimères hétérosexuées chez lesquelles les ovaires inhibés se développent après l'ablation des testicules, présentaient les caractères externes de la maturité sexuelle mâle et une hypertrophie des canaux de Wolff au moment de l'intervention. Au cours des mois suivants, les caractères externes régressent et I'hypertrophie des canaux de Wolff s'atténue légèrement, par contre les canaux de Müller jusque là non stimulés, s'hypertrophient dès que la vitellogenèse débute au niveau des ovaires. Cependant la stimulation du tractus müllérien lorsqu'elle est tardive, n'est jamais aussi importante que chez les femelles témoins. II est difficile d'invoquer ici une action inhibitrice émanant des testicules, telle que par exemple l'action d'une hormone anti-müllérienne comme chez les Vertébrés supérieurs, en effet le tractus müllérien des chimères hermaphrodites pourvues de testicules à maturité sexuelle, est aussi stimulé que celui des femelles témoins. D'ailleurs, quelle que soit l'importance des testicules chez les chimères $\sigma \leftrightarrow$ \& âgées seulement de 1 ou 2 ans, il suffit d'un petit élément ovarien avec quelques ovocytes en vitellogenèse pour avoir une stimulation locale du canal de Müller. En 
absence de stimulation précoce, les canaux de Müller perdraient leur sensibilité à l'action hormonale des ovaires.

Sans pour autant pouvoir donner une interprétation tant sur le plan embryologique que sur le plan biochimique, il ressort d'une analyse en cours que les phénomènes rencontrés à propos des chimères hétérosexuées à corps double sont encore plus démonstratifs à propos des chimères hétérosexuées à corps simple. Dès à présent, nous pouvons indiquer que le même effet de position se retrouve avec l'existence de gonades intersexuées ou hermaphrodites ayant une partie antérieure testiculaire et une partie postérieure ovarienne. Mais surtout, des preuves statistiques et génétiques rendent encore plus évidente l'inversion du phénotype sexuel ovarien dans les combinaisons hétérosexuées résultant de la greffe en chimère chez le Pleurodèle.

En conclusion, les interactions entre glandes génitales de sexe différent chez les Pleurodèles chimères à corps double dépendent de la position relative des gonades avec pour conséquence une grande diversité dans la réalisation du phénotype gonadique. Dans l'association ovaires antérieurs- testicules postérieurs, l'effet free-martin est particulièrement important, il peut conduire à l'inhibition totale des ovaires antérieurs et même fréquemment à leur inversion en testicules. Dans l'association testicules antérieurs- ovaires postérieurs, l'effet free-martin est bien plus faible, l'inhibition ovarienne totale n'a jamais été observée et l'inversion du phénotype ovarien est exceptionnelle. Par contre, il peut s'établir une inhibition mutuelle ou un état d'équilibre entre les gonades de sexe différent, susceptible d'être rompu tardivement aussi bien en faveur des testicules antérieurs qu'en faveur des ovaires postérieurs.

Reçu en juin 1986

Accepté en juin 1986

Remerciements. - Ce travail a bénéficié de la seule aide financière de l'Université Pierre et Marie Curie.

\section{Références}

BURNS R. K., 1935. The process of sex transformation in parabiotic Amblystoma. III - Conversion of testis to ovary in heteroplastic pairs of $A$. tigrinum and A. punctatum. Anat. Rec., 63, 101129.

CHARLEMAGNE J., HOUILLON Ch., 1974. Analyse morphologique des chimères obtenues par greffes embryonnaires chez les Amphibiens Urodèles. J. Embryol. exp. Morphol., 31, 263-286.

COLLENOT A., GOUNON P., COLLENOT G., 1977. Aspects récents de l'étude de la différenciation sexuelle des Amphibiens. Etude de l'effet free-martin chez le Triton Pleurodèle. Mém. Soc. Zool. Fr., 41, Symp. L. Gallien, 31-42.

DOURNON C., 1977. Régulation du nombre de cellules germinales primordiales dans les larves chimères à corps double chez Pleurodeles waltiii Michah. (Amphibien Urodèle). Mém. Soc. Zool. Fr., 41, Symp. L. Gallien, 61-70.

DOURNON C., HOUILLON Ch., 1984. Démonstration génétique de l'inversion fonctionnelle du phénotype sexuel femelle sous l'action de la température d'élevage chez l'Amphibien Urodèle : Pleurodeles waltiii Michah. Reprod. Nutr. Dévelop., 24, 361-378. 
FOOTE C. L., 1964. Intersexuality in Amphibians, 233-279. In Intersexuality in vertebrates inc/uding man (AMSTRONG et MARSHALL, édit.). Acad. Press.

GALLIEN L., 1962. Evolution chez le Triton Pleurodeles waltiii des intersexués obtenus après traitement par I'hormone femelle. Bull. Biol. Fr. Belg., 96, 249-280.

GALLIEN L., 1973. Différenciation et organogenèse sexuelle des Métazoaires. Paris, Masson et Cie.

GOUJON P., 1974. Induction de la tolérance aux allogreffes de peau dans les chimères de l'amphibien urodèle, Pleurodeles waltlii Michah. J. Embryol. exp. Morphol., 32, 805-818.

HOUILLON Ch., 1956. Recherches expérimentales sur la dissociation médullo-corticale dans l'organogenèse des gonades chez le Triton Pleurodeles waltlii Michah. Bull. Biol. Fr. Belg., 90, 359 445.

HOUILLON Ch., 1964a. Chimères xénoplastiques entre les Urodèles Pleurodeles waltii Michah. et Triturus alpestris Laur. C.R. Acad. Sci. Paris, 258, 3901-3903.

HOUILLON Ch., 1964b. Nouvelles chimères xénoplastiques chez les Urodèles : combinaisons viables et létales. C.R. Acad. Sci. Paris, 258, 5725-5728.

HOUILLON Ch., 1965. Associations en chimères entre I'Axolotl et diverses espèces de Triton. C.R. Acad. Sci. Paris, 261, 246-248.

HOUILLON Ch., 1976. Différenciation sexuelle des chimères chez les Amphibiens Urodèles. Bull. Soc. Zool. Fr., 101, suppl. 4, 103-107.

HOUILLON Ch., 1977. Tractus uro-génital des chimères chez I'amphibien urodèle Triturus alpestris Laur. J. Embryol. exp. Morph., 42, 15-28.

HOUILLON Ch., CHARLEMAGNE J., 1971. Développement d'un double système de glandes génitales dans les chimères homoplastiques à corps double chez l'Amphibien Urodèle, Pleurodeles waltiii Michah. C.R. Acad. Sci. Paris, Sér. D, 272, 1546-1549.

HOUILLON Ch., CHARLEMAGNE J., GOUJON P., 1973. Hermaphrodisme des chimères allogéniques chez I'Amphibien Urodèle, Pleurodeles waltlii Michah. C.R. Acad. Sci. Paris, Sér. D, 276. 1617-1620.

JOST A., PERCHELLET J. P., PREPIN J., VIGIER B., 1975. The prenatal development of bovine freemartins, 392-406. In REINBOTH R., Intersexuality in the animal kingdom, SpringerVerlag. 